\title{
Zwei Empfehlungsgedichte von Johannes Gregor Macer Szepsius (ca 1530 - nach 1579)
}

František Šimon

(Pavol Jozef Šafárik University in Košice)

\begin{abstract}
The humanist writer Johannes Gregor Macer Szepsius (circa 1530 - after 1579) came from the small town of Moldava nad Bodvou in present-day Slovakia. He studied at the Faculty of Arts in Cracow, and he continued working in Cracow after completing his studies. He wrote typical Latin occasional poetry, but he also became interested in alchemy and natural sciences. One of his friends, the Swiss physician Anton Schneeberger (1530-1581) published a catalogue of Latin and Polish plant names, and Macer contributed with a commendation poem here. It is written similarly to the well-known fourth ode of the first book of Horace and it has two parts, the first inspired by the arrival of spring, while the subject of the second part is Schneeberger's book itself. In 1562 Schneeberger published a work on salt and a commendation poem by Macer again appears there. The epigram is written in elegiac distich and partly reflects the content of the treatise, but Macer also adds his own thoughts. Both poems are good examples of occasional poetry, in which the author presents himself as a poet and a natural scientist.
\end{abstract}

\section{Keywords}

Johannes Gregor Macer Szepsius; occasional poetry 
Der humanistische Autor Johannes Gregor Macer Szepsius kam aus der kleinen ostslowakischen Stadt Moldava nad Bodvou. Nach dem ungarischen Namen der Stadt Szepsi hat er den Beinamen Szepsius gewählt.

Gregor Macer studierte an der artistischen Fakultät der Universität in Krakau, die damals von vielen ungarischen Gebildeten besucht wurde. Dort wirkte er auch nach der Beendigung des Studiums, es gibt Evidenz für seine Lehrertätigkeit an der Krakauer Universität. ${ }^{1}$ Er interessierte sich für die Naturwissenschaften und die Alchemie, schrieb Gelegenheitspoesie. ${ }^{2}$ Sein größtes poetisches Werk heißt De vera gloria libellus. ${ }^{3}$ Die Hauptidee des Werkes ist, dass der wahre Ruhm der Ruhm Gottes ist und er verurteilt das Streben nach Reichtum, Lust, Schönheit und Macht. Macer nennt im Gedicht einzelne Berufe und macht deren Vertretern Vorwürfe wegen vieler Mängel. ${ }^{4}$ Er war in Kontakt mit bekannten Krakauer Gebildeten, Ärzten, besonders mit den Anhängern der Paracelsus-Lehre. Er hat auch die lateinische Übersetzung der Paracelsus-Schrift Archidoxae herausgegeben, die in Krakau im Jahre 1569 erschien. ${ }^{5}$

Einer von seinen Freunden war auch der Schweizer Arzt, Schüler von Conrad Gessner (1516-1565), Anton Schneeberger (1530-1581), der in Krakau lebte und dessen erste Frau die Tochter des berühmten Krakauer Arztes Johannes Antoninus Cassoviensis (1496-1548), der aus Košice (lat. Cassovia, dt. Kaschau) stammte, war. ${ }^{6}$ Schneeberger hat mehrere medizinische Fachschriften geschrieben und Macer hat fast zu allen mit einem kurzen poetischen Werk beigetragen.

Im Jahre 1557 veröffentlichte Schneeberger einen Katalog der lateinischen und polnischen Pflanzennamen. ${ }^{7}$ Als Beispiel diente dem Autor das Werk seines Lehrers Conrad Gessner, der 1542 eine viersprachige Pflanzennamenliste publizierte. ${ }^{8}$ Schneebergers Buch enthielt eine alphabetische Liste der lateinischen Pflanzennamen mit den polnischen Äquivalenten und es wird als erstes polnisches terminologisches botanisches Wörterbuch angesehen. ${ }^{9}$

Macer hat hier mit einem Empfehlungsgedicht beigetragen und im Namen des Gedichtes bekennt er sich zu „Imitation der vierten Ode des ersten Buches von Horaz“. ${ }^{10}$ Diese - zusammen mit anderen horazischen Oden - hat im 16-17. Jh. die Poesie mit dem Thema Jahreszeiten beträchtlich beeinflusst. ${ }^{11}$ Macer hat das Metrum des Vorbildes behalten, da die Ode in der vierten archilochischen Strophe geschrieben wurde. Die

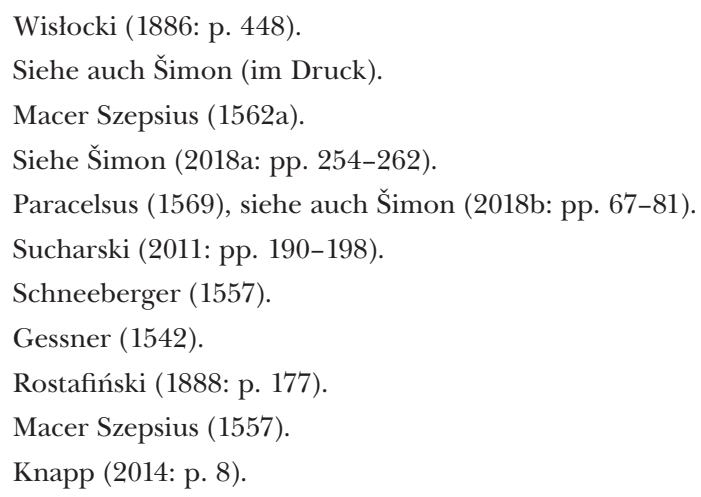


vierte horazische Ode hat zwei Teile, der erste ist ein Loblied an die Ankunft des Frühlings als Symbol der Wiedergeburt und im zweiten erinnert der Autor an die Vergebung des Lebens, den Tod, also an das Vergehen. Auch Macers Gedicht ist in zwei Teile gegliedert. Was die Verszahl betrifft, sind das ganz gleiche Teile, aber was den Inhalt betrifft, ist nur der erste von der Frühlingsankunft inspiriert, das Thema des zweiten Teiles ist das Buch Schneebergers selbst.

Wenn Horaz seine Ode mit dem bekannten „es löst sich der scharfe Winter“ anfängt, so hat Macer im zweiten Vers das gleiche Verb, aber im Partizip, „es rinnt der Strom des gelösten Schnees“.

solvitur acris hiems

Hor. Carm. 1.4.1

nivisque torrens labitur solutae

Macer In cat. ode 2

In Horaz soll das glänzende Haupt mit grüner Myrte bekränzt werden, in Macer streckt der neue Frühling das von den glänzenden Kränzchen bunte Haupt heraus.

viridi nitidum caput impedire myrto

verque novum nitidis pictum caput exerit corollis

Horaz sagt, dass im Frühling die Wiesen nicht mehr vom weißen Reif schimmern, Macer dagegen, dass prächtige Wiesen zu sehen sind.

nec prata canis albicant pruinis

Hor. Carm. 1.4.4

gramina purpureis pratis

Macer In cat. ode 9

Horaz beschreibt Tänze im Mondschein, während in Macer die warme Sonne scheint. imminente luna solis calor imminet

Hor. Carm. 1.4.5

Macer In cat. ode 1

Der zweite Teil des Gedichtes reflektiert den Fachinhalt des Buches, wobei wir gleich am Anfang erfahren, dass das Buch lateinische sowie polnische Pflanzennamen enthält. In beiden Teilen finden sich auch Anknüpfungen jeweils an den anderen Teil. In der Beschreibung des Frühlings sagt Macer, dass der Frühling auch neue Pflanzen mit sich bringt und der zweite Teil beginnt damit, dass auch Pflanzennamen kommen.

novasque secum (iam) reducit herbas

Macer In cat. ode 4 nomina graminibus veniunt quoque

Macer In cat. ode 11

Im dritten Vers streckt der neue Frühling das Haupt heraus, im dritten Vers vom Ende ist der Frühling für die Entstehung beider, d. h. Pflanzen sowie Bücher, geeignet.

verque novum caput exerit

Macer In cat. ode 3 utrisque ver est commodum creandis

Macer In cat. ode 18

Macer lobt natürlich den Autor und in einer Opposition spielt er auch mit seinem Namen. Einerseits schwindet der Schnee und die Bergketten beginnen zu grünen, andererseits strahlt Schneeberger mit den Blumen. 
Utque nives fugiunt, montis iuga floribus virescunt,

Schneebergius sic floribus nitescit

Macer In cat. ode 13-14

Macer spricht Schneeberger an, dessen Name bekränzt das Buch.

nomine tibi dedicato libellum ... coronas

Macer In cat. ode 15-16

\author{
Danach hebt Macer eine Parallele hervor, die Wiesen sind grün mit Pflanzen, und sie \\ sprossen in diesem Buch hervor. \\ Gramine prata virent, hoc gramina pullulant libello \\ Macer In cat. ode 17
}

Schließlich endet das Gedicht mit einem Vergleich, wie die Jungen (oder ein zartes Mädchen) die Pflanzen auf den Wiesen pflücken, so soll der Leser aus diesem Buch Belehrung gewinnen, wobei das Verb legere bzw. seine Ableitungen mehrmals vorkommen. Utque legunt iuvenes, pratis, tenera aut puella, flores

Sic lector ex hoc colligat libello

Macer In cat. ode 19-20

Im Gedicht kann man auch den Einfluss anderer horazischer Oden beobachten. Die Beschreibung des Winterwaldes in der neunten Ode des ersten Buches, nach der die leidenden Wälder die Last kaum ertragen, konnte für Macer Inspiration sein, weil für ihn die durch den Schnee erdrückten Bäume im Winter leiden.

(Soracte,) nec iam sustineant onus

silvae laborantes

Hor. Carm. 1.9.2-3 arbores decentes per vim nivosam dolebant

Macer In cat. ode 5-6

In der siebten Ode des vierten Buches ist der Schnee verschwunden, bei Macer verschwindet der Schnee ähnlich.

diffugere nives

Hor. Carm. 4.7.1

$$
\text { nives fugiunt }
$$

Macer In cat. ode 13

Macer konnte vielleicht auch anders zur Herausgabe dieser Arbeit beitragen. Der Autor Anton Schneeberger gibt in der Einleitung zu, dass er die polnische Sprache ungenügend beherrscht, was zu Überlegungen führt, wer ihm bei der Arbeit geholfen hat. Der ältere polnische Wissenschaftler J. Rostafiński nimmt an, dass es Johannes Antoninus der Jüngere (16. Jh.), in der Zeit der Arbeit sein zukünftiger Schwager, sein konnte, ${ }^{12}$ der später auch Schneebergers Pestschrift Buch über die Erhaltung der Menschengesundheit vor der Pestluft ins Polnische übersetzt hat. ${ }^{13}$ Es konnte aber auch sein guter Freund Macer

12 Rostafiński (1888: p. 177).

13 Schneeberger (1569). 
sein, kam er doch aus dem Gebiet der heutigen Slowakei, also aus einem slawischen Milieu. Umso mehr, da er auch möglicherweise nicht-ungarischer bzw. slowakischer Herkunft sein konnte. Die ungarischen Humanisten haben nämlich dies üblicherweise mit dem Beinamen Pannonius angedeutet ${ }^{14}$ und gerade diesen hat Macer auch in diesem Buch Schneebergers.

Im Jahre 1562 veröffentlicht Schneeberger eine Arbeit über das Salz und widmet sie der Erinnerung an seinen Schwiegervater Antoninus Cassoviensis. ${ }^{15}$ Der Autor entfaltet darin die vielen Vorteile von Salz in verschiedenen Bereichen des menschlichen Handelns und die Größe seines Nutzens wird durch Adjektive bestimmt. Einen gewissen Nutzen von Salz haben die Leute im Bauwesen und in der Metallverarbeitung, der Nutzen ist groß in der Landwirtschaft, sehr groß bei Lebensmitteln und der größte in der Medizin. Nach dieser Skala gibt Schneeberger diesen Bereichen eine entsprechende Textmenge.

Im Buch findet sich wieder Macers poetischer Beitrag, ein Gedicht, mit dem er dem Leser das Werk empfiehlt. ${ }^{16}$ Dieses Epigramm auf das Buch über den Nutzen von Salz ist im elegischen Distichon geschrieben und spiegelt teilweise den Inhalt der Schrift wider, aber Macer fügt auch seine eigenen Gedanken hinzu.

Das Gedicht beginnt mit einer gnomischen Aussage, dass, wenn etwas oft gefunden wird, die Leute weniger daran interessiert sind, obwohl es sich um viele Naturgeheimnisse handeln kann. Aber wenn etwas selten ist, erregt das Neugier, Lob und wird von allen für bewundernswert gehalten. Solch ein Fall ist auch eine Beschreibung des Salzes, die zum Erstaunen der „eifrigen Menge“ führt. Salz ist in den Mysterien des Neuen und des Alten Testaments gegenwärtig und gehört zu den Geheimnissen der Natur.

Der Autor als Anhänger der alchemistischen Lehre von Paracelsus, die mit Mysterien zusammenhängt, erwähnt in diesem kurzen Gedicht dreimal das Geheimnis, aber immer in einer anderen sprachlichen Form: abdita naturae, verborgene (Geheimnisse), Rätsel der Natur, mysteria legis veteris et novae, Mysterien des Neuen und des Alten Testaments, secreta (quae natura tegit), Geheimnisse (die die Natur verbirgt). Der Autor vergleicht Salz mit (Edel-)Steinen, Pflanzen, Edelmetallen, die dauerhaft sind.

Macer setzt dann mit den Themen der Arbeit selbst fort und fasst unter dem Begriff panacea die allseitigen von Schneeberger erwähnten Anwendungsmöglichkeiten von Salz in der Medizin zusammen. Im Schneebergers Buch selbst taucht allerdings ein solcher Name nicht auf.

Was schließlich die Lebensmittel betrifft, erwähnt er den Hauptnutzen des Salzes, den angenehmen Geschmack des Essens, den Salz liefert.

Macer als ein humanistischer Dichter ist zugleich auch ein Kenner der römischen Literatur, an einigen Stellen kommen mehr oder weniger wörtliche Entlehnungen meistens von Ovid vor. Aus den Dokumenten der Krakauer Universität wissen wir, dass er gerade

$\begin{array}{ll}14 & \text { Klaniczay (1993: pp. 108-109). } \\ 15 & \text { Schneeberger (1562). } \\ 16 & \text { Macer Szepsius (1562b: p. A3r). }\end{array}$ 
Metamorphosen gelesen hat. ${ }^{17}$

mirabile cunctis redditur

Macer In lib. ep. 3

sedula turba

Macer In lib. ep. 6

natura salis nomine clausa

Macer In lib. ep. 10 visum mirabile cunctis

Ov. Met. 12.165

sedula turba

Ov. Rem. 182

Tib. 1.4 .80

Caesareo nomine clausus

Ov. Fast. 1.282

Der ovidische Einfluss auf seinen Stil spiegelt sich auch in der Tatsache wider, dass das Ende seiner Distichen mit dem Ende des Satzes zusammenfällt.

Zusammenfassend kann man sagen, dass in dieser kleinen Probe Macers Schaffens Einfluss von zwei großen römischen Dichtern erkennbar ist, einerseits von Horaz, andererseits von Ovid. Beide Gedichte sind ein gutes Beispiel für Gelegenheitspoesie, in der der Autor sich als Dichter und Naturforscher präsentiert. Er verbindet Poesie mit einem professionellen Thema, in diesem Fall mit den Pflanzennamen, bzw. mit dem Salz als wichtiger Substanz für das menschliche Leben.

\section{Anhang}

In catalogum stirpium Anthonii Schnebergeri patritii Tigurini ODE Gregorii Macri Szepsii Pannonis, A[rtium] B[accalaurei] ad imitationem Quartae primi Horatii

Blandior auricomi Solis calor imminet sub(inde)

Nivisque torrens labitur solutae

Verque novum nitidis pictum caput exerit corollis,

Novasque secum iam reducit herbas.

5 Quasque comas gelidae Brumae, prius arbores dole(bant)

Per vim decentes, diripi nivosam

Iam modo frondiferi sperant ope Veris adfuturas,

Et se virenti iam coma novari.

Gramina purpureis pratis sua cernis advenire,

10 Quae flore terras imbuunt recenti

Nomina graminibus veniunt quoque redditis Lati(nis)

Polonicisque vocibus notata.

Utque nives fugiunt, montis iuga floribus virescunt,

Schneebergius sic floribus nitescit.

15 Nomine, ceu redoles flores, tibi dedicato, amoenos

17 Wisłocki (1889: p. 448). 
Sic hunc libellum floribus coronas

Gramine prata virent, hoc gramina pullulant libello

Utrisque ver est commodum, creandis.

Utque legunt iuvenes, pratis, tenera aut puella, flo(res)

20 Sic lector ex hoc colligat libello.

Gregorii Macri Szepsii in libellum De salis usu epigramma

Si quid forte frequens fuerit, vix cernitur ulli, naturae quamvis abdita multa premat.

Contra si quod adest rarum, mirabile cunctis redditur et laudes ex novitate refert.

5 Huius ab exemplo salis hic conscribitur usus, mirari ut possit sedula turba salem.

Sal veteris, pariterque novae mysteria legis

continet, et magnis addita sacra diis.

Quin etiam secreta docet quamplurima prudens

10 quae natura salis nomine clausa tegit.

Hinc si quidem lapides, plantae et preciosa metalla durant, purgantur, hincque colore nitent.

Nam neque flamma salem, nec destruit unda, vicissim in se quin redeat viribus ipse suis.

15 Sal fovet humani iustam quoque corporis aevo temperiem longo, ceu Panacea potens.

Adde cibos, quorum, nisi sal praestasset amoenum gustum, corporibus noxius usus erat.

Ne tamen hic multis lector teneatur, in isto

20 plura legens cernet, quam putat ipse libro.

\section{Bibliographie}

Gessner, C. (1542). Catalogus plantarum latine, graece, germanice et gallice. Tiguri: apud Christoph. Froschouerum.

Klaniczay, T. (1993). Die Benennungen „Hungaria“ und „Pannonia“ als Mittel der Identitätssuche der Ungarn. In T. Klaniczay, S. K. Németh, \& P. G. Schmidt (Eds.), Antike Rezeption und nationale Identität in der Renaissance insbesondere in Deutschland und in Ungarn (pp. 83-110). Budapest: Balassi kiadó.

Knapp, É. (2014). „Volucris rota, vertitur anni“: Zrínyi Miklós, Listius László és Esterházy Pál szerencse- és évszakverseinek poétikatörténeti hátteréhez. Irodalomtörténeti Közlemények, 118, 3-30.

Macer Szepsius, G. Io. (1557). In Catalogum Stirpium Anthonii Schnebergeri Ode. In A. Schneeberger, Catalogus stirpium quarundam Latine et Polonice conscriptus (p. A4r). Cracoviae: Lazarus Andreae. 
Macer Szepsius, G. Io. (1562a). De vera gloria libellus. Cracoviae: Lazarus Andre[a]e.

Macer Szepsius, G. Io. (1562b). In libellum de salis usu epigramma. In A. Schneeberger, De multiplici salis usu (p. A3r). Cracoviae: Lazarus Andreae.

Paracelsus, Ph. Th. (1569). Archidoxae libri X. Cracoviae: ex officina Mathiae Wirzbietae.

Rostafiński, J. (1888). Nasza literatura botaniczna XVI wieku, oraz jej autorowie lub tlumacze. Pamiętnik Academii Umiętności w Krakowie. Wydzial matematyczno-przyrodniczy, 14, 152-207.

Schneeberger, A. (1557). Catalogus stirpium quarundam Latine et Polonice conscriptus. Cracoviae: Lazarus Andreae.

Schneeberger, A. (1562). De multiplici salis usu. Cracoviae: Lazarus Andreae.

Schneeberger, A. (1569). Ksiazkki o zachowaniu zdrowia człowieczego od zarazy morowego powietrza. Krakow: u Mattheußa Siebbeneychera.

Sucharski, R. (2011). Antoni Schneeberger i Jan Grzegorz Macer - dwaj przyjaciele w szesnastowiecznym Krakowie. In L. Buzássyová, E. Juríková, \& N. Sipekiová (Eds.), Decus Sapientiae. Sambucus Supplementum (Vol. III; pp. 190-198). Trnava: Towarzystvo Slowakóv w Polsce, Filozofická fakulta Trnavskej univerzity.

Šimon, F. (im Druck). Neznáme diela humanistu Jána Gregora Macera Szepsia (1530 - po 1579) [Unbekanntes Werk von Johannes Gregor Macer Szepsius (1530 - nach 1579)]. Sambucus, 12.

Šimon, F. (2018a). Satira na povolania v diele J. G. Macra Szepsia De vera gloria libellus [Satire auf die Berufe in Werk von J. G. Macer Szepsius De vera gloria libellus]. In L. Buzássyová, \& E. Juríková, Ideológia v premenách času v pamiatkach gréckej a latinskej tradície (pp. 254-262). Bratislava: Univerzita Komenského v Bratislave.

Šimon, F. (2018b). Úvod J. Gregora Macera Szepsia k Paracelsovmu dielu Archidoxae (1569) [Einleitung zu Paracelsus' Werk Archidoxae (1569) von J. Gregor Macer Szepsius]. Kultúrne dejiny / Cultural history, 9, 67-81.

Wisłocki, W. (1886). Liber diligentiarum facultatis artisticae Universitatis Cracoviensis Pars I (14871563). Cracoviae: F. Kluczycki.

doc. PhDr. František Šimon, CSc. / frantisek.simon@upjs.sk

Department of Classical Philology

Pavol Jozef Šafárik University in Košice, Faculty of Philosophy

Moyzesova 9, 04001 Košice, Slovak Republic 